\title{
ON THE EXTENSION OF LINEARLY INDEPENDENT SUBSETS OF FREE MODULES TO BASES
}

\author{
BYOUNG-SONG CHWE AND JOSEPH NEGGERS
}

Introduction. In this note we discuss a class of rings with identity with the following property:

(1) Each linearly independent subset of a (unitary) free right $A$-module can be extended to a basis, by adjoining elements of a given basis. In view of (1) we call such rings right-Steinitz rings. We prove the equivalence of (1) and the following condition:

(2) Let $R_{1}=\{x \in A \mid x$ does not have a left inverse $\}$. If an infinite matrix $T$ of elements of $R_{1}$ is column-finite and if $T_{i j}=0$ for all $i \leqq j$, then, for each $j$, there is an integer $N$ such that $\left(T+T^{2}+\cdots+T^{n}\right)_{j+n, j}$ $=0$ for all $n>N$.

To prove the equivalence of (1) and (2) we need to establish several other properties of right-Steinitz rings, which in turn reveal them as being either examples or "near-examples" of classes of rings studied by a variety of investigators, the following cases being representative. In [1], P. M. Cohn discusses a sequence of three progressively stronger conditions, the strongest being

III. Any generating set with $n$ elements of a rank $n$ free module is free. An inductive argument shows that right-Steinitz rings do indeed satisfy the condition. It also follows from the discussion below that right-Steinitz rings satisfy all conditions of Goldie's local-rings except that the intersection of all powers of the ideal of nonunits may not be zero (cf., e.g., [2]). Obviously, division rings are right-Steinitz rings. If $Z$ is the ring of integers and if $p$ is any prime, then $Z /\left(p^{i}\right)$ satisfies condition (2) as is easily seen. For any field $\Delta$ and a vector-space $V$ over $\Delta$, let $A=\Delta \times V$, with operations defined by

$$
\begin{aligned}
\left(\delta_{1}, x_{1}\right)+\left(\delta_{2}, x_{2}\right) & =\left(\delta_{1}+\delta_{2}, x_{1}+x_{2}\right) \\
\left(\delta_{1}, x_{1}\right)\left(\delta_{2}, x_{2}\right) & =\left(\delta_{1} \delta_{2}, x_{1} \delta_{2}+x_{2} \delta_{1}\right), \quad \delta_{i} \in \Delta, \quad x_{i} \in V .
\end{aligned}
$$

Then, $V$ is the ideal of nonunits, with $V^{2}=0$, and again condition (2) is easily seen to be satisfied. Another property of right-Steinitz rings is the following: if $\left\{x_{i}\right\}_{i=0}^{\infty}$ is a sequence of nonunits, then, for some index $n, x_{n} \cdot x_{n-1} \cdots x_{1}=0$. Thus, let $F_{0}$ be a division-ring, and let $F_{0}[x]$ be the polynomial-ring in one variable over $F_{0}$. Define $F_{i}$ $=x F_{0}[x] / x^{i+1} F_{0}[x]$ for $i \geqq 1$. Let $R$ be the weak direct sum of rings

Presented to the Society, January 23, 1969; received by the editors September 25, 1968. 
$F_{i}$. Then, $R$ is a two-sided vector-space over $F_{0}$. Take $A=F_{0} \times R$, with operations

$$
\begin{aligned}
\left(\delta_{1}, x_{1}\right)+\left(\delta_{2}, x_{2}\right) & =\left(\delta_{1}+\delta_{2}, x_{1}+x_{2}\right), \\
\left(\delta_{1}, x_{1}\right) \cdot\left(\delta_{2}, x_{2}\right) & =\left(\delta_{1} \delta_{2}, x_{1} \delta_{2}+\delta_{1} x_{2}+x_{1} x_{2}\right), \quad \delta_{i} \in F_{0}, \quad x_{i} \in R .
\end{aligned}
$$

Notice that if $x_{0}=\left(\alpha, \cdots, \alpha_{i+1}, 0, \cdots, 0, \cdots\right) \in R$, then given any sequence $\left\{x_{i}\right\}_{i=0}^{\infty}$ of elements in $R, x_{i} \cdot x_{i-1} \cdot \cdots \cdot x_{0}=0$. From this, again, condition (2) follows. Notice that in this case the ideal $R$ is not nilpotent, while if $R$ is nilpotent, (2) follows easily.

Clearly, if $T$ is an infinite proper triangular matrix, i.e., a triangular matrix with 0 diagonal, over any ring, then the inverse of $I-T$ exists and is equal to $I+T+T^{2}+\cdots$. The argument depends on the fact that $I-T$ as well as $I+T+T^{2}+\cdots$ are row finite and because $\left(T^{n}\right)_{i j}=0$ if $n>i-j$. We can thus restate condition (2) to obtain the equivalent form:

$(2)^{\prime}$ If $T$ is an infinite column-finite proper triangular matrix of elements of $R_{1}$, so is $(I-T)^{-1}$. In concluding this introduction we should like to thank the referee for several helpful comments and a simplification of the proof of Theorem 2.

The equivalence of conditions (1) and (2). Note that all modules under discussion are right unitary.

Lemma 1. If $A$ satisfies (1), then for each infinite sequence $\left\{x_{i}\right\}_{i=0}^{\infty}$ of elements of $A$ which do not have a left inverse, there is a nonnegative integer in such that $x_{n} x_{n-1} \cdots x_{0}=0$.

Proof. Let $\left\{u_{i}\right\}_{i=0}^{\infty}=U$, be a basis for a free $A$-module $M$, i.e $M=[U]=\left[U_{i}\right]_{i=0}^{\infty}$. Let $v_{i}=u_{i}-u_{i+1} x_{i}, \quad i=0,1,2, \cdots$ Then, $\left\{v_{i}\right\}_{i=0}^{\infty}$ is linearly independent. Indeed, $\sum_{i=0}^{s} v_{i} a_{i}=0$ implies $\sum_{i=0}^{s}\left(u_{i}-u_{i+1} x_{i}\right) a_{i}=0$, i.e.,

$$
u_{0} a_{0}+u_{1}\left(a_{1}-x_{0} a_{0}\right)+\cdots+u_{s}\left(a_{s}-x_{s-1} a_{s-1}\right)-u_{s+1} x_{s} a_{s}=0,
$$

whence $a_{0}=a_{1}=\cdots=a_{s}=0$. Now let $V$ be the submodule spanned by $\left\{V_{i}\right\}_{i=0}^{\infty}$. Since $\left\{V_{i}\right\}_{i=0}^{\infty}$ can be extended to a basis of $M$ by adjoining elements of $U$, suppose $\left\{v_{i}\right\}_{i=0}^{\infty} \cup\left\{u_{i_{1}}, u_{i_{2}}, \cdots\right\}$ is a basis of $M$. Then $u_{i_{1}} \equiv u_{i_{2}} y(\bmod V)$ for some $y \in A$ if $i_{1}<i_{2}$, whence $u_{i_{1}}$ $\in \operatorname{span}\left(v,\left\{u_{i_{2}}\right\}\right)$. Thus $\left\{v_{i}\right\}_{i=1}^{\infty} \cup\left\{u_{t}\right\}$ must be a basis for some $u_{t} \in U$. Then, $u_{t+1} \equiv u_{t} a(\bmod V), u_{t} \equiv u_{t+1} x_{t}(\bmod V)$. Hence, $u_{t} \equiv u_{t} a x_{t}$ $(\bmod V)$, i.e., $1-a x_{t}=0$. Since $x_{t}$ does not have a left inverse, $V=M$, and $\left\{v_{i}\right\}_{i=0}^{\infty}$ is a basis of $M$. Thus, if $\sum_{i=0}^{s} v_{i} b_{i}=u_{0}$, i.e.,

$$
u_{0} b_{0}+u_{1}\left(b_{1}-x_{0} b_{0}\right)+\cdots+u_{\theta}\left(b_{s}-x_{s-1} b_{s-1}\right)-u_{s+1} x_{s} b_{s}=u_{0},
$$


we have $b_{0}=1, b_{1}=x_{0}, \cdots, b_{8}=x_{8-1} \cdots x_{0}, x_{8} b_{8}=x_{8} \cdots x_{0}=0$. Hence, $n=n(s)=s$ and the lemma follows.

Lemma 2. Let $A$ be a ring with identity, $R_{1}=\{x \in A \mid x$ does not have a left inverse $\}$ and $R_{2}=\{x \in A \mid x$ does not have a right inverse $\}$. If every element of $R_{1}$ is nilpotent then $R_{1}=R_{2}$ and $R_{1}$ forms the unique maximal ideal of $A$.

Proof. First we show that $R_{1}^{c}=A \backslash R_{1}$ forms a group. It is clear that $R_{1}^{c}$ is closed under multiplication. Suppose $x \in R_{1}^{c}$, then there is a $y \in A$ such that $y \cdot x=1$. If $y \notin R_{1}^{c}$ then there is an integer $n$ such that $y^{n}=0$, $y^{n-1} \neq 0$. Hence, $0=y^{n} \cdot x=y^{n-1}$, and this is a contradiction. So, $y \in R_{1}^{c}$. Therefore, if $y x=1$ then $x y=1$. Thus, $R_{1}^{c} \subset R_{2}^{c}$. Hence $R_{1} \supset R_{2}$. Suppose $x \in R_{1}$ and $x \notin R_{2}$, then there is a $y \in A$ such that $x y=1$. Since $x$ is nilpotent, this is also a contradiction. Hence $R_{1}=R_{2}$. To show $R_{1}$ is closed under + , let $x$ and $y$ be elements of $R_{1}$, and suppose $x+y \in R_{1}$. Then there is a $z \in A$ such that $z(x+y)=1, z x+z y=1, z x=1-z y$. Since $z y \in R_{1}$, it is nilpotent and $1-z y$ has an inverse, i.e., $z x$ has an inverse. This is a contradiction. Hence $R_{1}$ is closed under + . It is clear that $z R_{1} \subset R_{1}$ for any $z \in A$. Also if $x \in R_{1}, z \in A$ and $z x \in R_{1}$, then there is a $y \in A$ such that $y x z=1$. This is a contradiction because $y x$ is nilpotent. Hence $R_{1}$ is an ideal of $A$. It is clear that $R_{1}$ is the unique maximal ideal of $A$ because $R_{1}^{c}$ consists of the units of $A$. In short, since $R_{1}$ forms a left ideal, Lemma 2 follows as is well known.

Corollary 3. If, for each infinite sequence $\left\{x_{i}\right\}_{i=0}^{\infty}$ of elements of $A$ which do not have a left inverse there is a nonnegative integer $n$ such that

$$
x_{n} \cdot x_{n-1} \cdots x_{0}=0 \text {, }
$$

then there is a nonzero element $a$ of $A$ such that $b \cdot a=0$ for all elements $b$ of $A$ which do not have a right inverse.

Proof. Let $R_{2}$ be the collection of all nonzero elements of $A$ which do not have a right inverse. If, for all nonzero elements $x$ of $R_{2}, R_{2} x$ $\neq\{0\}$, we have a choice function $f: R_{2} \backslash 0 \rightarrow R_{2} \backslash 0$, such that $(x) f \cdot x \neq 0$, whence each nonzero $x_{1}$ in $R_{2}$ generates an infinite sequence $\left\{x_{1}, \cdots, x_{n}, \cdots\right\}$ with $x_{i}=\left(x_{i-1} \cdots x_{1}\right) f$, such that $x_{n} \cdot x_{n-1} \cdots x_{1}$ $\neq 0$ for each integer $n$. This is a contradiction. Hence, since $R_{2}=\{0\}$ implies $R_{2} \cdot 1=0$, the lemma follows.

Theorem 1. If a ring $A$ satisfies (1), then it satisfies (2).

Proof. From Lemma 1 and Lemma 2, $A$ has a unique maximal ideal consisting of all nonunits, $R_{1}=R_{2}=R$. Let $T$ be a matrix provided by (2), and let 


$$
u=\left\{u_{j} \mid j=1,2, \cdots\right\}
$$

be a basis of a free $A$-module $M$. Let

$$
v_{j}=u_{j}-\sum_{l} u_{l} T_{l j} \quad \text { for } j=1,2,3, \cdots,
$$

then clearly $V=\left\{v_{j} \mid j=1,2,3, \cdots\right\}$ is a linearly independent subset of $M$. From the corollary to Lemma 2 , there is a nonzero element $a$ such that $R a=0$. Hence $v_{j} a=U_{j} a$ for each $j$. Since we suppose that $A$ satisfies (1) and $V$ is a basis of $M$. Suppose $\sum_{j=1}^{i+n} v_{j} S_{j i}=u_{i}$ and $S_{j i} \in A$ for each $i$. Let $S$ be the matrix whose elements are $S_{j i}$, then $(I-T) S$ $=1$ where $I_{i j}=\delta_{i j}$, where as mentioned before, $S=I+T+T^{2}+\cdots$.

Lemma 3. Let $A$ be a ring satisfying condition (2), then for each sequence $\left\{x_{i}\right\}_{i=1}^{\infty}$ of elements of $R_{1}$, there is an $n$ such that

$$
x_{n} \cdot x_{n-1} \cdots x_{1}=0 \text {. }
$$

Proof. Consider the case $T_{i j}=x_{j}$ if $i=j+1$ and $T_{i j}=0$ if $i \neq j+1$. Then

$$
\left(T^{n}\right)_{n, 1}=T_{n+1, n} \cdot T_{n, n-1} \cdots T_{2,1}=x_{n} \cdot x_{n-1} \cdots x_{1} .
$$

Hence from condition (2), $x_{n} \cdot x_{n-1} \cdots x_{1}=0$.

Lemma 4. If $A$ satisfies condition (2) then $R_{1}=R_{2}=R$ and if $R \neq\{0\}$ there is a nonzero element $a \in R$ such that $R a=0$, and $R$ is the unique maximal ideal from Lemmas 3, 2 and the corollary to Lemma 2.

Lemma 5. Let $A$ be a ring as in the corollary to Lemma 2, then any finite linearly independent subset of a free $A$-module $M$ can be extended to a basis by adjoining elements of a given basis.

Proof. Let $V=\left\{v_{1}, v_{2}, \cdots, v_{n}\right\}$ be a linearly independent set, and $U=\left\{u_{i} \mid i \in \Lambda\right\}$ be a basis of $M$. Let $v_{1}=\sum u_{i} a_{i}$ for $a_{i} \in A$, then not all $a_{i}$ are elements of $R$, otherwise $v_{1} a=\sum u_{i} a_{i} a=0$, where $a$ is the element of $A$ of Corollary 3. Let $a_{1} \notin R$, then $u_{1}=\left(v_{1}-\sum_{i>1} u_{i} a_{i}\right) a_{1}^{-1}$, hence $\left\{v_{1}\right\} \cup\left\{u_{i} \mid i \neq 1\right\}$ is a basis. Suppose $\left\{v_{1}, v_{2}, \cdots, v_{n-1}\right\}$ $\cup\left\{u_{i} \mid i>n\right\}$ is a basis and $v_{n}=\sum_{i<n} v_{i} b_{i}+\sum_{i \geq n} u_{i} a_{i}$, then not all $a_{i}$ are in $R$, otherwise $v_{n} a=\sum_{i<n} v_{i} b_{i} a$. Hence $v_{1}, v_{2}, \cdots, v_{n}$ can be extended to a basis by adjoining some elements of $U$. Therefore, by induction, the lemma is proved.

Theorem 2. If a ring $A$ satisfies (2) then it satisfies (1).

Proof. Let $U=\left\{u_{i} \mid i \in \Lambda\right\}$ be a basis of $M$, and $V=\left\{v_{j} \mid j \in T\right\}$ be a linearly independent subset. Without loss of generality we may 
assume that $V$ is a maximal linearly independent subset of $V \cup U$. Suppose $[V] \neq M$, then there is a $u_{1} \notin[V]$. Let $u_{1} c=\sum_{j=1}^{n} V_{j} b_{j 1}$ for some $c \in A$ and $b_{j 1} \in A$. Since $\left\{v_{1}, v_{2}, \cdots, v_{n}\right\}$ can be extended to a basis by adjoining some elements of $U$,

$$
u_{1}=\sum_{j} v_{j} b_{j 1}^{\prime}+\sum_{l} u_{l} T_{l 1} \quad \text { for some } b_{j 1}^{\prime}, \quad T_{l 1} \in A,
$$

whence $b_{j 1}^{\prime} C=b_{j 1}$ and $T_{l 1} C=0$ for all $j$ and $l$. Hence $T_{l 1} \in R$ and $u_{1} \equiv \sum_{l \geqq 1} u_{l} T_{l 1} \bmod [V]$. If $T_{11} \neq 0$, then $u_{1} \neq \sum_{l \geqq 2} u_{l} T_{l 1}\left(1-T_{11}\right)^{-1}$ $\bmod [\mathrm{V}]$, and we may thus assume $u_{1} \equiv \sum_{l_{32}} u_{l} T_{l 1} \bmod [V]$. Repeating this argument, we obtain a countably infinite column-finite matrix $T$ of elements of $R$ such that $T_{l i}=0$ if $l \leqq i$ and $u_{i} \equiv \sum_{l} u_{l} T_{l i}$ $\bmod [V]$. By $(2)^{\prime}, S=(I-T)^{-1}$ is column-finite. If $X$ denotes the row matrix $\left(u_{1}, u_{2}, \cdots\right)$, then $X(I-T) \equiv 0 \bmod [V]$ implies $(X(I-T)) S$ $\equiv 0 \bmod [V]$, contradicting the fact that $u_{1} \notin[V]$.

Corollary. If a ring $A$ satisfies (2), then, for any $A$-module $M$, $M=M R$ implies $M=\{0\}$.

Proof. Let $\left\{u_{i} \mid i \in T\right\}$ be a generating set, then for each $u_{i}, u_{i}$ $=\sum_{l} u_{l} T_{l i}$ where $T_{l i} \in R_{1}$. We can assume that $T=\{1,2, \cdots\}$ and $T_{l i}=0$ if $l \geqq i$ as before. Then, $u_{i}-\sum_{l} u_{l} T_{l i}=0$ implies $u_{i}=0$ for each $i$.

\section{REFERENCES}

1. P. M. Cohn, Some remarks on the invariant basis property. Topology 5 (1966), 215-228. MR 33 \#5676.

2. A. W. Goldie, A note on noncommutative localization, J. Algebra 8 (1968), 41-44. MR 36 \#6442.

The University of Alabama 